\title{
Study on the differences of opinions and choices of high-risk breast cancer populations in China before and after genetic testing
}

\author{
Xiaolin Cheng, Zhangyuan Gu, Xiaoyin Sun, Zhigang Zhuang \\ Department of Breast Surgery, Shanghai First Maternity and Infant Hospital, Tongji University School of Medicine, Shanghai 201204, China \\ Contributions: (I) Conception and design: X Cheng, Z Zhuang;(II) Administrative support: Z Zhuang; (III) Provision of study materials or patients: \\ X Cheng; (IV) Collection and assembly of data: XCheng, X Sun; (V) Data analysis and interpretation: X Cheng, Z Gu, Z Zhuang; (VI) Manuscript \\ writing: All authors; (VII) Final approval of manuscript: All authors. \\ Correspondence to: Zhigang Zhuang. Department of Breast Surgery, Shanghai First Maternity and Infant Hospital, Tongji University School of \\ Medicine, Shanghai 201204, China. Email: zhuang_zg@163.com.
}

\begin{abstract}
Background: In recent years, genetic testing (GT) has developed rapidly in China. However, the cancer genetic service system is still immature in China. Little is known about the opinions around genetic counseling (GC) and GT in Chinese individuals at high risk of breast cancer. This work aims to understand the changes in awareness, attitudes, and willingness of high-risk populations before and after GT, as well as the possible barriers to GC/GT.
\end{abstract}

Methods: Several questionnaires for the high-risk populations of breast cancer were completed. Then, some patients were selected for free single/multi-gene testing as well as further survey and statistical analysis. Results: Despite low levels of awareness, Chinese high-risk breast cancer groups hold positive attitudes about GC/GT. There were differences in the willingness of different groups of high-risk people. After GT, different testing results led to different views and choices for the patients than before GT. Lack of genetic knowledge of breast cancer, misunderstanding, cost, fear of adverse effects, and policy market factors are obstacles to accepting GC/GT for the participants.

Conclusions: Chinese doctors need to communicate according to the specific situation of high-risk individuals in pre-test counseling and post-test counseling. The national policy and GT market also need to be improved to support the hereditary breast cancer families' follow-up service system.

Keywords: Genetic counseling (GC); genetic testing (GT); hereditary breast cancer; Chinese; questionnaire

Submitted Jun 07, 2019. Accepted for publication Oct 11, 2019.

doi: $10.21037 /$ tcr.2019.11.43

View this article at: http://dx.doi.org/10.21037/tcr.2019.11.43

\section{Introduction}

Breast cancer is one of the most frequently diagnosed cancers globally and is the leading cause of cancer death in women (1). Up to $10 \%$ of breast cancers are due to specific mutations in single genes that are passed down in a family (2-5). The recent forty years of cancer registry data document a tremendous increase in incidence for breast cancer among women in Shanghai (6). Moreover, some patients are significantly impacted by their genetic inheritance (7). The field of cancer genetics has implications for all aspects of cancer management of individuals with hereditary or familial cancers, including prevention, screening, and treatment (8). At present, the mutation spectrum for these susceptibility genes is not well understood in the Chinese population, and there are few reports on prevention and clinical intervention in high-risk populations (7).

In 2018, AstraZeneca's Lynparza (olaparib) was approved in China, for the therapy of ovarian and breast cancer 
Table 1 Entry criteria

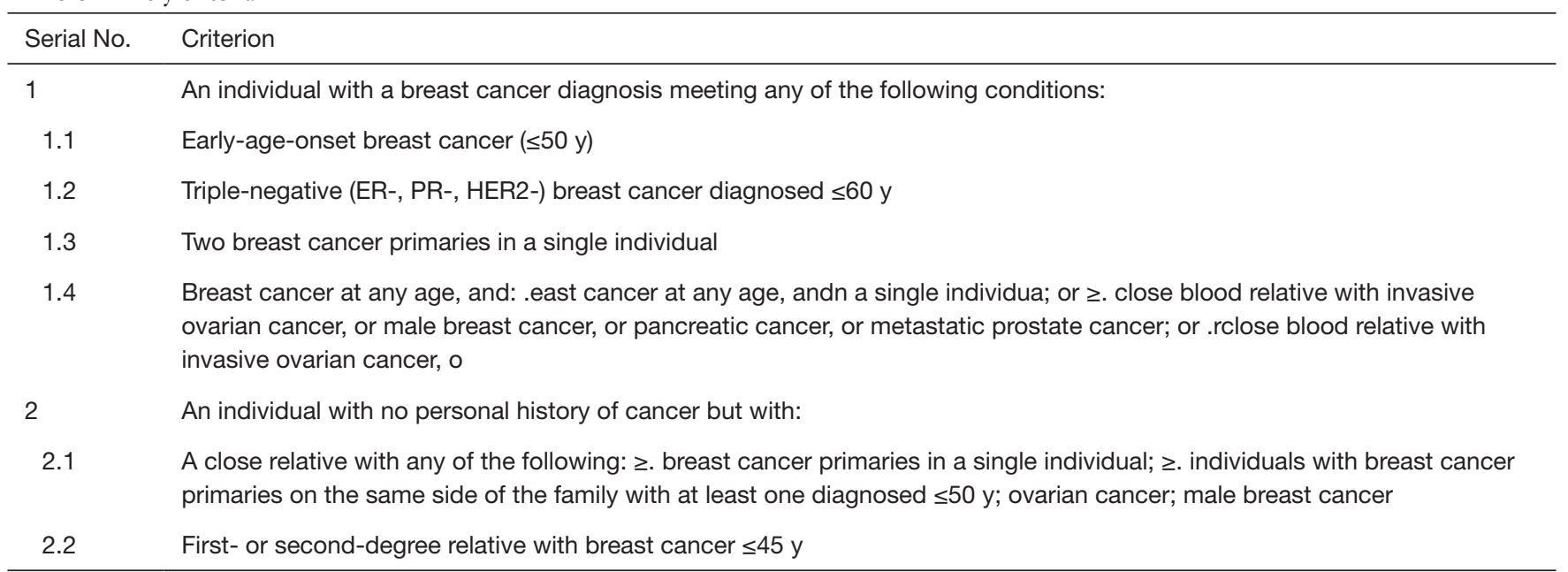

patients with the BRCA1/2 mutation (9). In clinical practice, both doctors and patients are more concerned about the BRCA1/2 (breast cancer 1/2) gene than other genes, which have also been studied more in China (10-12). The NCCN (The National Comprehensive Cancer Network) Guidelines Panel has already added information regarding multi-gene testing for the 2014 update. Multigene testing may be most useful when more than one gene can explain an inherited cancer syndrome, and this multitesting may also be more efficient and cost-effective $(13,14)$. However, multi-gene testing for breast cancer related to inherited cancer syndrome is rarely studied in China (7).

Many patients undergoing genetic testing (GT) do not receive proper pre-test and post-test GC. Previously, we conducted research on the knowledge and willingness of breast cancer patients and their relatives to genetic counseling (GC) and GT $(15,16)$, but in our recent clinical work, it was found that the opinions and attitudes of the high-risk groups were very different from those ordinary cases; furthermore, after GT, the opinions and choices of high-risk populations also changed. It is well-known that in China, few studies focus on the high-risk groups' perceptions and attitudes during the GC and GT processes. Also, the high-risk groups and families have not been well screened, managed, and advised.

Therefore, this study included 1,349 individuals genetically at a high-risk for breast cancer to statistically analyze and understand their knowledge about GC and GT. Specifically, we assessed the willingness to choose, barriers, and attitudes before and after GT, in addition to exploring the barriers and motivators of electing GC and GT.

\section{Methods}

\section{General information}

After obtaining approval from Shanghai First Maternity and Infant Hospital and Ethics Committee (IRB number KS1412), between the years January 2015 and December 2017 , a total of 1,349 patients who met the study conditions, along with their blood relatives, completed the anonymous survey in the Department of Breast Surgery in Shanghai First Maternity and Infant Hospital (the entry criteria are shown in Table 1). Documented informed consent form was obtained from each participant for future use of her samples and personal information for breast cancer-related studies. Also, patients who were illiterate or under the age of 18 , or had ever undergone breast cancer GT, were excluded from participating in the study. The classification of respondents is shown in Table 2.

\section{Survey details}

The survey included an information page which provided a simple explanation of hereditary breast cancer, GC, single and multi-gene testing, questions to obtain information regarding age, education, fertility, income, etc. (see Table 2), and "yes or no" survey questions about awareness, willingness, attitudes, and possible barriers, etc. to GC and GT (see Table 3). A total of 839 eligible cases were collected in the outpatient waiting room, and another 510 eligible forms were returned from the participants' relatives, which were filled out at their home. 
Table 2 Demographics of survey participants

\begin{tabular}{|c|c|}
\hline Characteristic & $\mathrm{n}(\mathrm{N}=1,349)$ \\
\hline \multicolumn{2}{|l|}{ Age at diagnosis (yrs.) } \\
\hline$\leq 50$ & 583 \\
\hline$>50$ & 766 \\
\hline \multicolumn{2}{|l|}{ Education } \\
\hline No college degree & 1,089 \\
\hline College degree & 260 \\
\hline \multicolumn{2}{|l|}{ Fertility } \\
\hline Have a daughter & 859 \\
\hline Not have daughter & 490 \\
\hline \multicolumn{2}{|l|}{ Place of birth } \\
\hline City-born & 368 \\
\hline Country-born & 981 \\
\hline \multicolumn{2}{|l|}{ Family income/year } \\
\hline$<\$ 30 \mathrm{~K}$ & 654 \\
\hline$\geq \$ 30 \mathrm{~K}$ & 695 \\
\hline \multicolumn{2}{|l|}{ Breast cancer history } \\
\hline Only personal history & 265 \\
\hline Only family history & 751 \\
\hline Personal and family history & 333 \\
\hline \multicolumn{2}{|l|}{ Recruitment method } \\
\hline Fill out the form at the clinic & 839 \\
\hline Return the form from home & 510 \\
\hline
\end{tabular}

\section{GT}

The GT is performed by using next-generation-sequencing technology (this test cannot detect balanced translocation, large rearrangements, inversions, ploidy changes, uniparental disomy, and methylation alterations).

From each family, the one with the highest probability of mutation was selected as the candidate. Only when the candidate satisfied 2 or more items in Table 1, was a free BRCA1/2 gene test given (due to the limited funding for the study).

\section{Survey after gene testing}

(I) The BRCA1/2 tester was given pre-test counseling and post-test counseling and completed the questionnaire (see Table 4).

(II) Those who participated in the free BRCA1/2 and in whom no mutation was found, were, if they so wished, given free multi-gene testing, pre-test counseling, and post-test counseling, and completed the post-test questionnaire (see Table 5).

\section{Statistical method}

The survey was classified according to age, family income, education, breast cancer history, and the statistical data were descriptive. The results of the questionnaire were counted in the form of "yes or no", and the answers were recorded in the form. For each question, the comparison between the data of each classification was analyzed by using the chisquare test. If the conditions for the chi-square test were not met (for example, if the frequency was too small), the Fischer's exact test was used. Fischer's exact test was used between the two sets of data for each classification. The statistical difference between the groups was based on the ratio of "yes" for each question, using a $95 \%$ confidence interval. Data analysis was performed using $\mathrm{R}$ language software (version 3.5.1).

\section{Results}

\section{Survey before GT (see Table 6)}

Overall, before GT, the high-risk genetic population knew extraordinarily little about cancer GC and GT, so the awareness rate for each was only $6.1 \%$ and $16.8 \%$ respectively. However, there was a strong willingness to accept GC and GT, with an $86.4 \%$ and $63.8 \%$ rate, respectively. Among high-risk individuals, $85.8 \%$ were willing to accept free BRCA1/2 GT while only $36.1 \%$ were willing to pay for it (the rate was as low as $4.3 \%$ in unaffected individuals). Meanwhile, $88.7 \%$ of high-risk individuals were willing to accept free multi-gene testing, while $62.3 \%$ were willing to pay for multi-gene testing (the corresponding rate was $75.5 \%$ in unaffected individuals). Most individuals in the high-risk groups were willing to share their GT results to help their families (78.1\%).

(I) The younger group had more knowledge about GC and GT and was more willing to accept GC and GT. They were also more worried about the possible adverse effects of the genetic test results, resulting in a weaker willingness to share their GT results. 
Table 3 Survey questions before GT

\begin{tabular}{ll}
\hline No. & Question \\
\hline 1 & Did you know about genetic counseling before today? \\
2 & Would you be willing to have genetic counseling? \\
3 & Did you know about genetic testing before today? \\
4 & Would you be willing to have genetic testing? \\
5 & If the test results are positive, are you worried about the negative impact? \\
6 & If it is good for the prevention and treatment of breast cancer in the future, would you be willing to spend about $\$ 500$ on the \\
7 & $\begin{array}{l}\text { BRCA1/2 gene test for your families? (Informing that the relevant, targeted drug Olaparib will be listed in China) } \\
8\end{array} \quad \begin{array}{l}\text { If it is good for your family and your prevention of breast cancer, would you be willing to spend about } \$ 800 \text { on multi-gene testing } \\
\text { for your families? }\end{array}$ \\
9 & If it is good for your family and your prevention of breast cancer, would you like free multi-gene testing for your families? \\
10 & Would you like to share your GT results to help your families?
\end{tabular}

GT, genetic testing.

Table 4 Questionnaire after BRCA1/2 test

\begin{tabular}{ll}
\hline No. & Question \\
\hline 1 & $\begin{array}{l}\text { If it is good for prevention and future treatment, would you like to inform the first-degree relatives who are not sick? (Explain first- } \\
\text { degree relatives) }\end{array}$ \\
2 & If it is good for prevention and future treatment, would you like to tell other non-first-degree relatives who are not sick? \\
3 & Are you worried that the test results will have a negative impact? \\
4 & Are you willing to recommend your family members to participate in self-pay genetic testing? \\
5 & Are you willing to recommend your family members to participate in the cancer screening program? \\
6 & Are you willing to enter the free multi-gene testing process? \\
\hline
\end{tabular}

Table 5 Questionnaire after multi-gene testing

\begin{tabular}{ll}
\hline No. & Question \\
\hline 1 & If it is good for prevention, would you like to inform the first-degree relatives who are not ill? (Explain first-degree relatives) \\
2 & If it is good for prevention, would you like to tell other non-first-degree relatives who are not ill? \\
3 & Are you worried that the test results will have a negative impact? \\
4 & Are you willing to recommend your family members to participate in self-pay genetic testing? \\
5 & Are you willing to recommend your family members to participate in the cancer screening program? \\
\hline
\end{tabular}

(II) The highly educated group did not have more knowledge of GC and GT than their counterparts. They had a stronger willingness to accept GC, but a weaker willingness to accept GT and more of them worried about the negative impact of the genetic test results. They were less likely to choose GT and share GT results with families (whether single-gene or multi-gene testing, and whether 


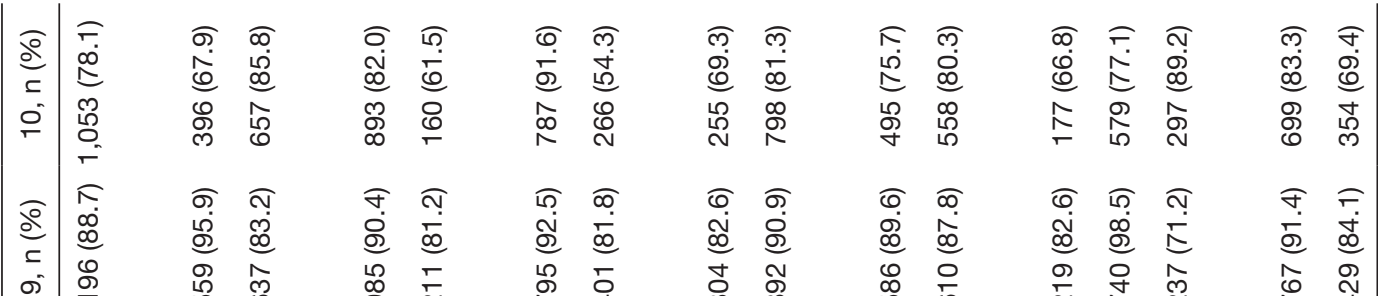

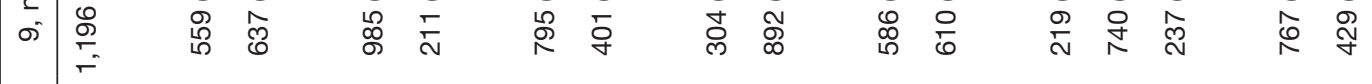

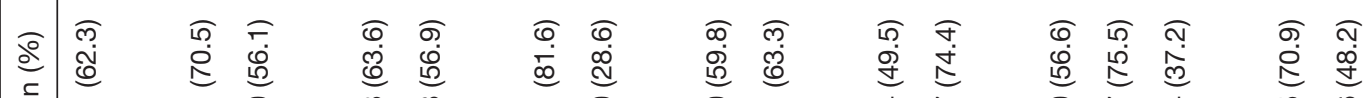

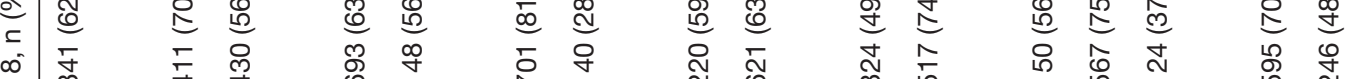

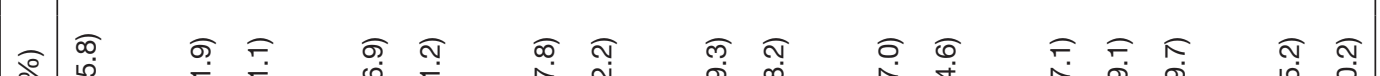

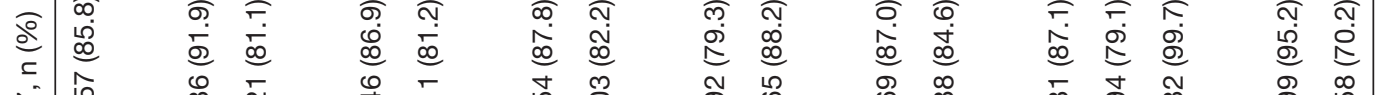

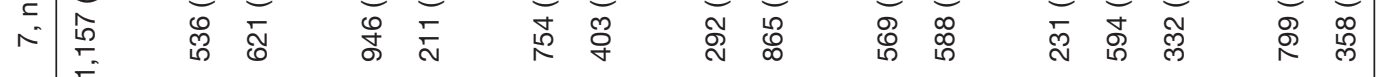

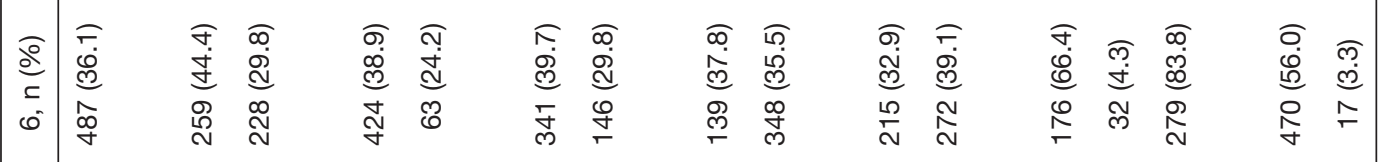

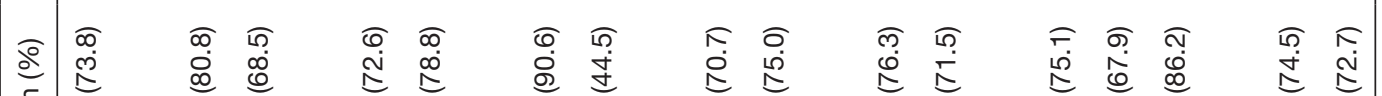

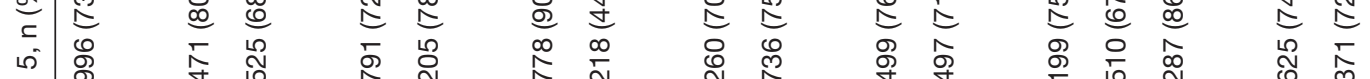

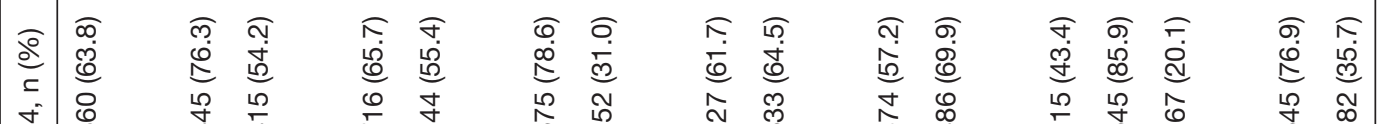

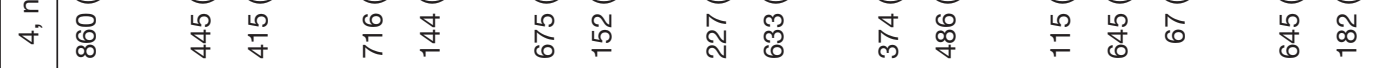

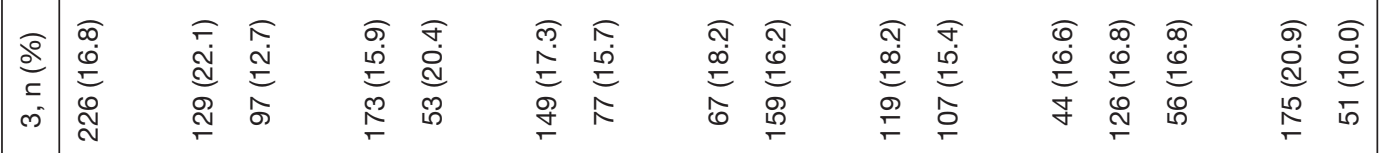

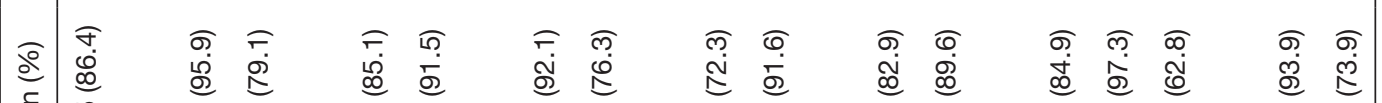

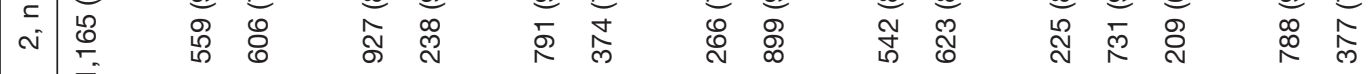

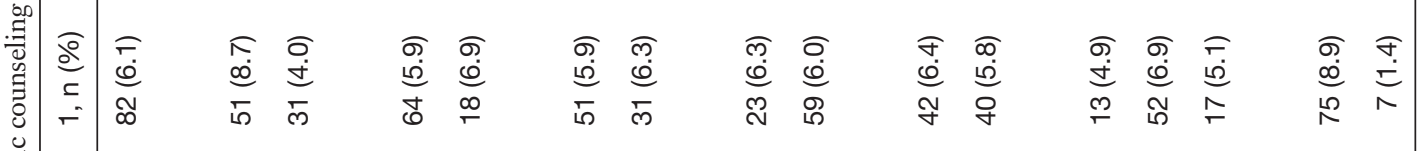

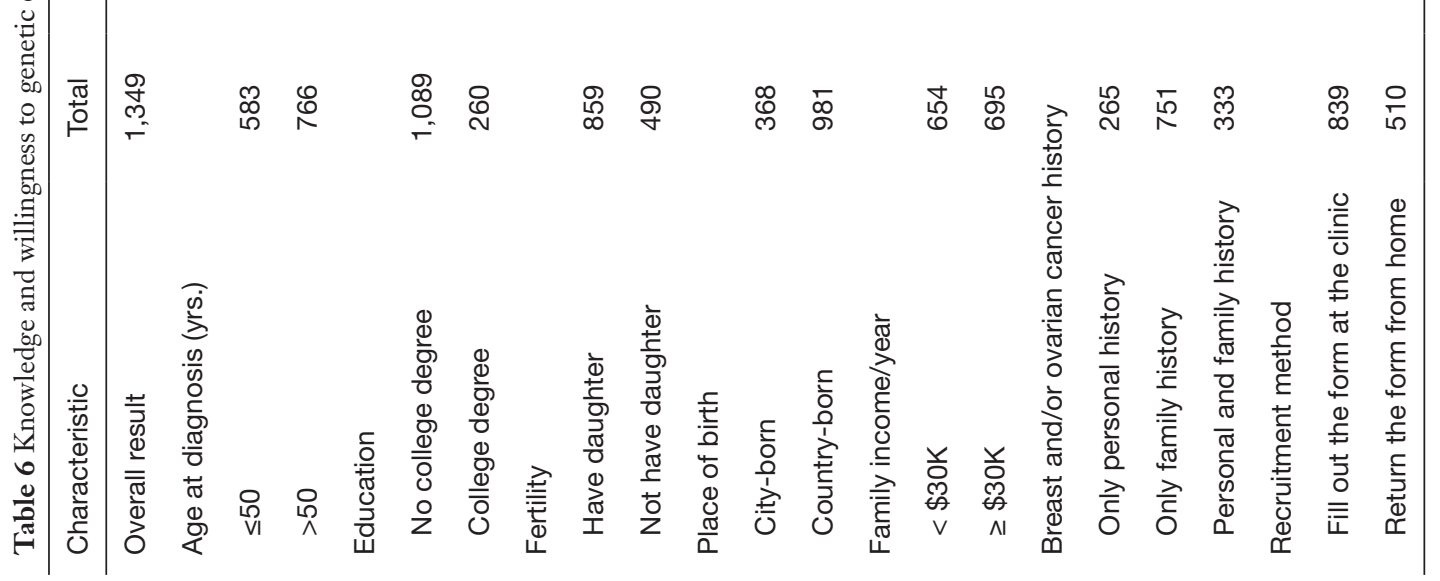


being charged for this service or not).

(III) The high-risk group with daughters had a higher willingness to receive GC and GT (whether in single-gene or multi-gene testing, and whether being charged for this service or not), and were more willing to share their GT results to help their families, but were more worried about the possible adverse impact from the genetic test results.

(IV) The high-risk populations born in rural areas were more willing to accept GC, more willing to participate in free GT, and more willing to share their GT results to help their families.

(V) High family-income groups were more willing to accept GC and GT, and more likely to accept self-funded GT. Also, they were more willing to share their GT results. Meanwhile, the low familyincome groups were more worried about the adverse effects of the genetic test results.

(VI) The groups with a family history of cancer (but not a personal one) were most willing to accept GC and GT. Also, they had the highest willingness to accept multi-gene testing for prevention (whether being charged or not); however, they had the lowest willingness to accept single-gene testing for future treatment (whether being charged or not). They worried least about the adverse effects of the genetic test results. The group with both personal and family history of cancer had the lowest willingness to receive GC and GT. Whether being charged for the service or not, they had the lowest willingness to accept multi-gene testing for future prevention, but the highest willingness to accept single-gene testing for future treatment. They were the most concerned about the adverse consequences of the genetic test results. The groups with only a personal history of cancer (but not a family one) had an intermediate willingness to accept GC and GT (whether in single-gene or multi-gene testing, and whether being charged for this service or not), and worried about the adverse consequences of the test results the second most. The groups with both personal and family history of cancer had the strongest willingness to share their GT results with their families, the group with only a family history was the second most willing, and the group with only a personal history had the lowest willingness.

(VII) The high-risk population who completed the questionnaire in the outpatient clinic was more aware of GC and GT and was more willing to accept them (whether in single-gene or multi-gene testing, and whether being charged for this service or not). They had a stronger willingness to accept GT and were more willing to share their GT results to help their families.

\section{Survey after single GT (see Table 7)}

A total of 359 people were eligible and willing to participate in single GT, and 316 people finally finished the free detection of BRCA1/2, yielding a rate of $88.0 \%$. The results showed 56 cases of pathogenic variants and 7 cases of likely pathogenic variants with a total mutation rate of $19.9 \%$.

The patients with negative results of BRCA1/2 gene test were $100 \%$ willing to tell their first-degree relatives and $75.9 \%$ willing to tell other relatives. Among these patients, those aged, country-born, and with both a personal and family history were more willing to inform other non-firstdegree relatives. For those with positive results, only $58.7 \%$ would tell the first-degree relatives, and $27 \%$ would tell other relatives. Those who had daughters and were countryborn were more willing to inform first-degree relatives and were worried about the negative impact of the result. The country-born patients were more willing to inform other relatives. The patients with negative results did not worry about the adverse effects of the results, while $93.7 \%$ of the patients with positive results were worried.

Furthermore, $42.9 \%$ of the patients that tested positive for BRCA1/2 GT were willing to recommend family members to a self-pay BRCA1/2 GT, among whom, those who had daughters and had a personal and family history were more willing to participate. The patients with positive BRCA1/2 results were $100 \%$ willing to recommend their families to the cancer screenings. For those receiving a negative result for the BRCA1/2 test, $93.3 \%$ were still willing to recommend families to cancer screenings, among whom, those who had daughters, were country-born, or had a personal and family history had a stronger willingness. A total of $81.0 \%$ of the BRCA1/2 mutation-negative patients were willing to accept further free multi-gene testing to find other genes that may be mutated. Those with daughters, those who were country-born, those with a personal and family history, and those who filled in a form in the clinic were more willing to accept further multi-gene testing. In contrast, the BRCA1/2 mutation-positive patients had a lower willingness to accept free multi-gene testing (33.3\%), 


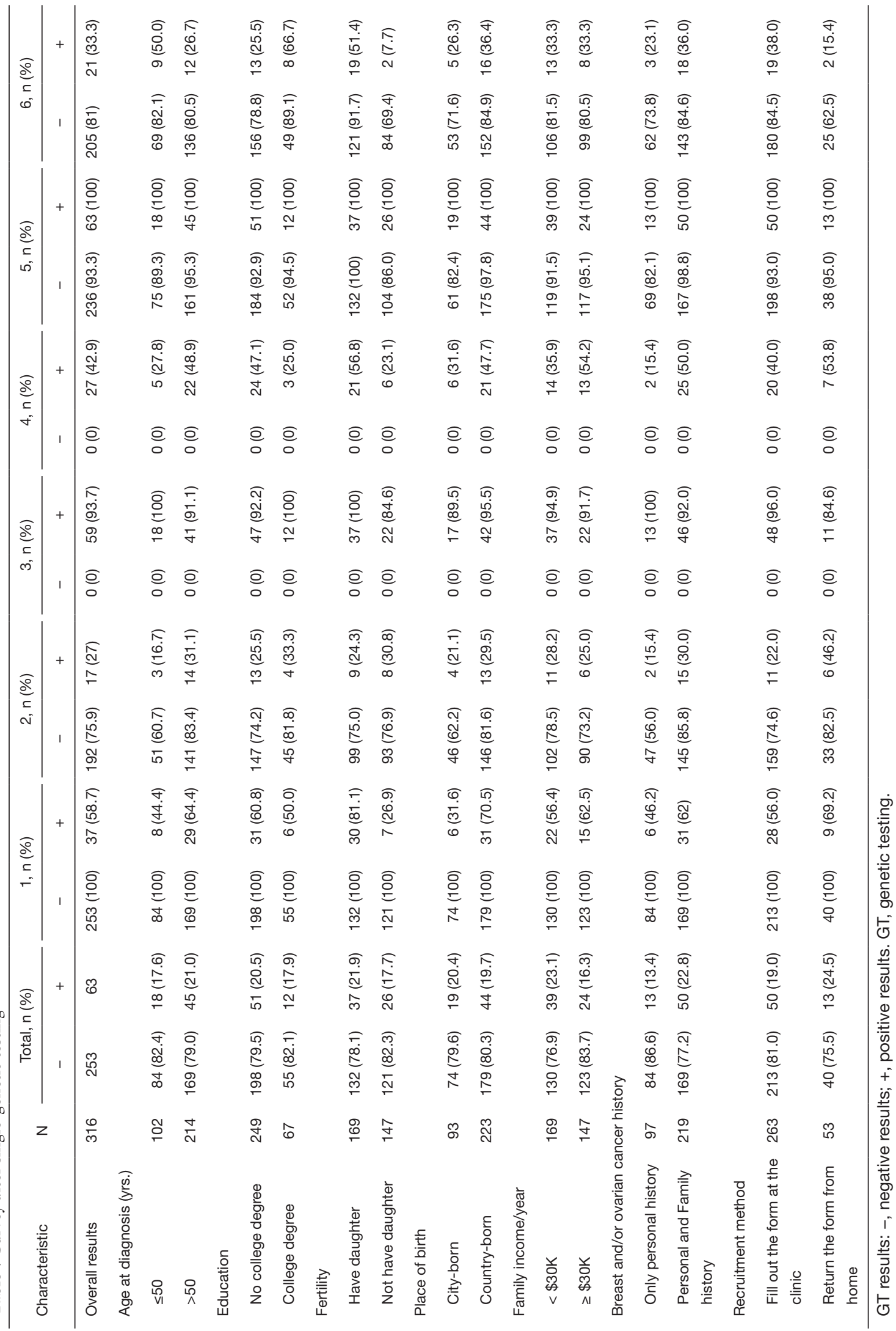


while the patients with high education and with daughters were more willing to accept further multi-gene testing.

\section{Survey after multi-gene testing (Table 8)}

A total of 205 people were eligible and were willing to accept multi-gene testing, and 162 people accepted the GT $(79.0 \%)$. Finally, 19 positive results were found with a mutation rate of $11.7 \%$.

Similar to the single-gene testing, in the multi-gene testing, the patients with negative results were more willing to inform relatives, with a $100 \%$ willingness to tell firstdegree relatives and an $81.1 \%$ willingness to tell other non-first-degree relatives. Meanwhile, for those with positive results, only $57.9 \%$ were willing to tell the firstdegree relatives, $15.8 \%$ were willing to tell other relatives, and $100 \%$ worried about the negative impact (such as discrimination) of the testing results. None of the patients with negative results were willing to recommend their family members to pay for GT, and only $10.5 \%$ of patients with positive results were willing to recommend their family members. On the other hand, $91.6 \%$ of patients with negative results and $100 \%$ of patients with positive results were willing to recommend family members to participate in the cancer screening program.

For the individuals in whom no mutation was found, those aged or country-born were more willing to inform their non-first-degree relatives; those aged, with daughter, country-birth, or with personal and family history were more willing to recommend their family members participate in the cancer screening program; no one worried about the adverse effects.

In those individuals with mutations, there were no significant differences found in the survey questions; this was due to the small sample size.

\section{Comparison before and after GT (see Table 9)}

Before GT, $81.3 \%$ of patients were worried about the adverse effects (such as discrimination) of positive results. After GT, the worrying rate increased for those with positive results $(93.7 \%$ and $100 \%$ for single-gene testing and multi-gene testing, respectively).

Before GT, $77.1 \%$ of the high-risk patients were willing to use their testing results to help the families. However, after GT, the willingness of those with positive results decreased significantly both for single-gene testing (27\%) and multi-gene testing (15.8\%).
Before GT, $76.1 \%$ of the high-risk patients were willing to pay for their family's single-gene testing. After GT, the willingness weakened considerably for those with positive results $(42.9 \%)$.

Similar to single-gene testing, for multi-gene testing after GT, the willingness rate of self-pay for families markedly decreased when the patients had positive multigene testing results (from $45.8 \%$ to $10.5 \%$ ).

\section{Discussion}

GC and GT for hereditary breast cancer have the potential benefit of early detection and early intervention. In modernday China, there is no systematic screening management for those with an elevated risk of hereditary breast cancer. There are several problems to be solved to improve genetic cancer services in clinical practice for breast cancer. The Shanghai First Maternal and Infant Health Hospital was founded in February 1947. It was one of the earliest specialized hospitals for treating female diseases in China, and its patient population hospital can represent the state of the medical community of the large cities in China.

Our study found that most of the high-risk groups were unaware of the knowledge about breast cancer inheritance, even those with higher levels of education. However, they had a strong willingness to accept GC and GT. The willingness of high-risk populations varied greatly before and after GT, and the individuals themselves were not aware of their change in attitude. In many cases of those tested, the original intention was focused on cancer prevention for their families. However, when facing positive GT results, they began to be averse about telling their families. When conducting GC, clinicians should be made aware of the patients' psychological changes before and after GT, and fully evaluate and skillfully guide the patients to better facilitate the prevention and treatment of the families and the patients themselves.

Younger people are more exposed to the Internet and therefore know more information about GC. For example, the Angelina Jolie gene detection incident, has, through a celebrity effect, inadvertently encouraged GT and GS (17). The younger group in our study did have a more positive willingness to choose GC and GT. However, the young group showed low willingness to share their GT results with their families, due to the pressure of young women in employment, mate selection, and issues of privacy. On the other hand, younger patients usually believed that since no older individual in the family was ill, that the disease 


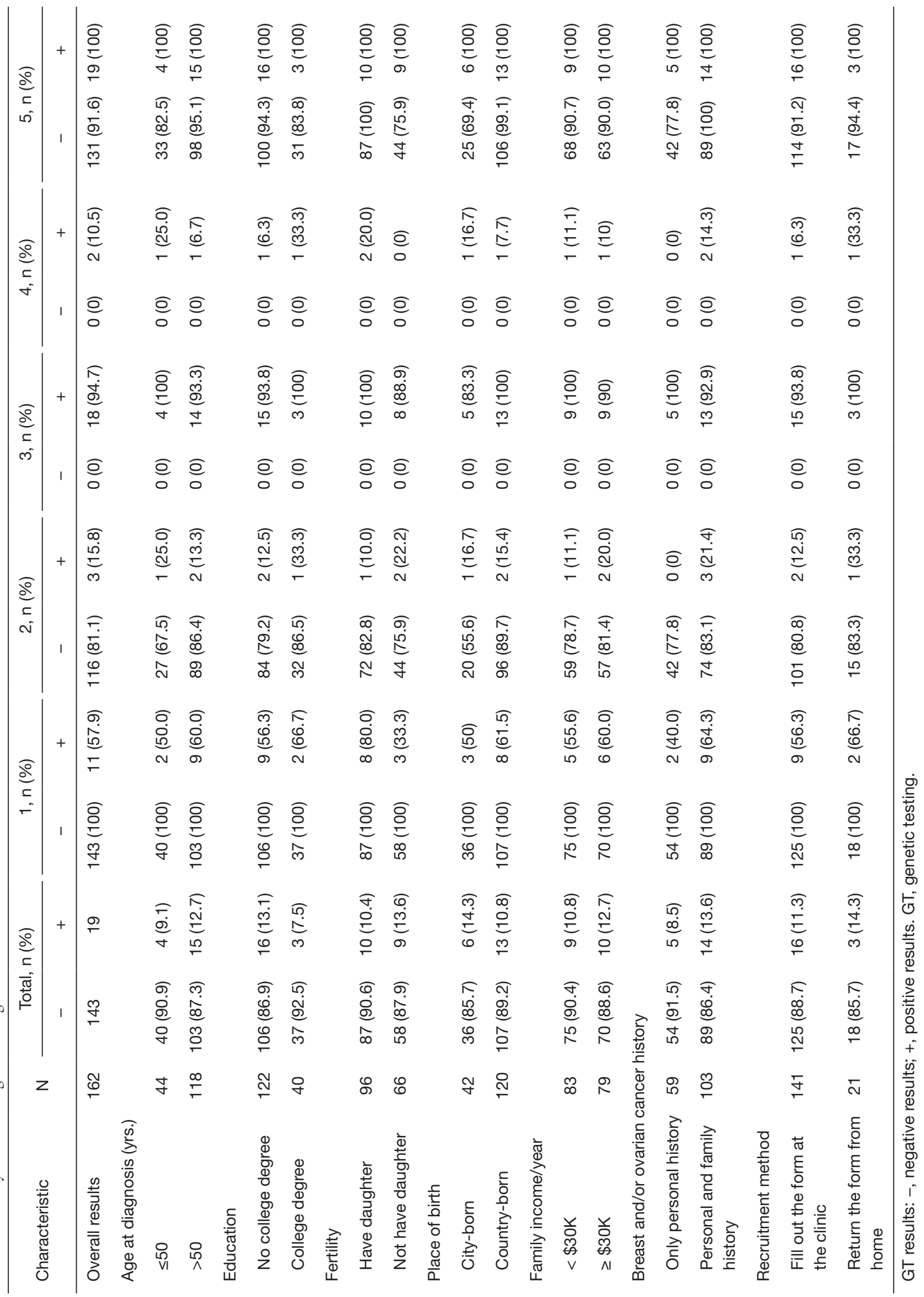


Table 9 Comparison before and after GT

\begin{tabular}{|c|c|c|c|c|c|}
\hline Characteristic & $\begin{array}{c}\text { Patients before } \\
\text { GT, n (\%) }\end{array}$ & \multicolumn{2}{|c|}{ After single-gene testing, $\mathrm{n}(\%)$} & \multicolumn{2}{|c|}{ After multi-gene testing, $\mathrm{n}(\%)$} \\
\hline Worried about being discriminated against & $486(81.3)$ & $0(0)$ & $59(93.7)$ & $0(0)$ & $19(100)$ \\
\hline Sharing GT results to help families & $579(77.1)$ & $192(75.9)$ & $17(27.0)$ & $116(81.1)$ & $3(15.8)$ \\
\hline Self-pay single-gene testing & $455(76.1)$ & 0 & $27(42.9)$ & - & - \\
\hline
\end{tabular}

GT results: -, negative results; +, positive results. GT, genetic testing.

was in no way heredity. This misunderstanding needs to be dispelled: the other family members may carry diseasecausing mutations but may have not yet developed the disease; final onset and time of onset are determined by a combination of factors without regard to age order $(3,17,18)$. Women diagnosed with breast cancer at an early age are more likely to carry a cancer-predisposing genetic mutation.

The biggest motivation of many high-risk individuals participating in this research was to learn about their own genetic status to benefit their daughter. However, when the results returned positive, they become hesitant and worried about the adverse effects, and a portion of these women chose not to tell their daughters the results. It might have been that the original intent of these patients was to confirm a negative result, so the testers with negative results were $100 \%$ willing to inform their first-degree relatives, while only about half that percentage of patients were willing to do so in the group with positive results. The high-risk groups without daughters often believe that their offspring are not genetically at risk. Thus, the following clarification must be made to them: breast cancer oncogene mutations can also be passed on to males and cause disease. BRCA1/2 mutations can increase prostate cancer, breast cancer, pancreatic cancer, and incur other risks for men (18). Some hereditary syndromes share several features beyond the elevation of breast cancer risks like Li-Fraumeni syndrome (LFS), Cowden syndrome, and hereditary diffuse gastric cancer (HDGC) which are related to germline mutations in the TP53, PTEN, and CDH1 genes, respectively (19-21). These syndromes arise from germline gene mutations that are not within sex-linked genes; hence, the mutations can be inherited from either parent.

Unexpectedly, the participants who were unaffected by cancer had more interest in GC and GT than breast cancer survivors. Before GT, the non-patients in high-risk groups were more likely to have "cost-effective" self-pay multi- gene testing, while the patients were more likely to have BRCA1/2 single-gene testing related to their treatment. In contrast to their opinions before GT, after testing, the patients with positive results were unwilling to disclose their genetic status to their families and were not willing to recommend their families to have self-pay GT for future prevention.

The unaffected women usually wanted to perform GT on their own, rather than recommending priority testing for an affected family member. It is best to consider testing an affected family member first, especially a family member with early-onset disease, bilateral disease, or multiple primaries because that individual has the highest likelihood for a positive test result. Women with personal and family history often avoid GC/GT, as they are convinced that they must be hereditary cancers families and do not need GC/ GT. They need to be reminded that familial cancers share some but not all features of hereditary cancers. Familial breast cancers do not exhibit the inheritance patterns or onset age consistent with hereditary cancers. For example, although familial breast cancers occur in a given family more frequently than in the general population, they generally do not exhibit the inheritance patterns or onset age consistent with hereditary cancers. Familial cancers may be associated with chance clustering of sporadic cancer cases within families, genetic variation in lower penetrance genes, a shared environment, or combinations of these factors $(2,3)$.

Affected individuals in the family should have priority GT, and the cost is lower for this testing. The selection of appropriate candidates for GT is based on the personal and familial characteristics that determine the individual's prior probability of being a mutation carrier, and on the psychosocial degree of readiness of the person to receive genetic test results. For the patient with characteristics of early-onset, bilateral, triple-negative, multiple tumors, etc. reminding them of the possible high genetic risk (22-25) of 
GC/GT is recommended even if there are no other patients in her family. Exploring different types of information needs among affected versus unaffected women can be a critical area for future research to inform targeted efforts to improve knowledge about GC/GT among different types of women who would benefit from GC/GT. The patients can benefit more from being given different measures to preventive treatment by identification of familial cancers and hereditary cancers with GC/GT.

There are still discrepancies in the number of people who are willing to have multi-gene testing and who finally perform the test. After experiencing a free BRCA1/2 test, they may think more and are feel not adequately prepared to undergo further testing. Breast cancer patients usually think that they do not need multi-gene testing since they already have cancer. Even if we explain that the genetic mutations may also increase the risk of other cancers and that they need preventive screening, they still refuse to face the harsh possibility of having other cancers. Although their original intention is to facilitate better prevention for their family members, their mentality might change after the test, even if repeatedly told that, for individuals with positive results, their first-degree relatives still have a $50 \%$ possibility of being GT negative. The test can eliminate the unnecessary anxiety of the relatives and avoid excessive screening or even pathological biopsy.

There are still many patients with positive results who are unwilling to tell their family members, choose to avoid them, or even refuse to contact them. This cannot be attributed to the indifference of the tested group since most participants still have a willingness to recommend their relatives to participate in screening and monitoring even with negative results. Many women feel that their relatives are already doing everything they can to minimize their risk of developing breast cancer; they are not willing to face the confirmed genetic mutation. On the one hand, they fear the emotional toll of finding out that they are a mutation carrier, especially if they have children who would be at risk of inheriting the mutation. On the other hand, GC and GT are not routinely carried out in hospitals and are not covered by medical insurance, and they are not willing to aggravate the mental and financial burden of the family. There is a need to inform them that regular breast cancer screening is not enough, and to make them aware of the clinical implications of mutation status for not only early breast cancer management but also for the patient's own and family members' cancer risk. For example, carriers of BRCA1/2 mutations not only have an increased risk of breast cancer but also have an increased risk of ovarian cancer. Ovarian cancer is often not easy to diagnose early, the disease progresses rapidly, and the prognosis is poor. Therefore, the effective prevention of fallopian tube oophorectomy in individuals with disease mutations can significantly reduce the risk of illness (26).

In the past 30 years, due to China's urbanization process, public medical resources continue to lean toward big cities. For example, many people born in rural areas come to Shanghai to live and seek medical treatment (27). Chinese rural-born population used to gather and live in their parents' home, and they have closer interactions with their relatives compared with those born in urban areas. However, as a new immigrant population, their economic levels are usually relatively low. In targeting specific population characteristics, the doctor needs to understand the patient's relevant genetic, medical, psychosocial, and personal information and be able to integrate this information before they can make an informed decision. Also, in all aspects of the groups of outpatient filling are more willing to take the initiative. How to encourage other family members to participate in the outpatient clinic is worthy of further study. Genetic counselors should provide additional guidance to counselees with serious cancer worry, emotional distress, a high-risk perception, or a weak social network (28).

Barriers of carrying out GC and GT include a poor understanding of the GT and GC, fear of carrying the mutation, concerns about discrimination, and cost. The GT application policy has not been liberalized, the GT market is not yet mature, and some high-risk individuals with high education have more doubts about the limitations of GT technology. The improvements in state policy and the market are necessary for the success of GC/GT. In 2018, Olaparib was approved in China, for the treatment-related single gene. The patients have a stronger willingness to have self-pay BRCA1/2 single-gene testing. On the other hand, both doctors and patients have little knowledge of other inherent cancer genes. Clinicians do not adequately communicate with the patients about how multi-gene testing will benefit their families.

Selection bias is a possible limitation of this study. Between the groups who are willing to complete the survey and those who are unwilling to complete the survey, there might be inherent differences in the results. Since in each family, only one member was tested for free, the results of the surveys after testing are limited and may not represent all high-risk individuals. Also, there might be 
differences between the free-of-charge group and the selffunded group. In other words, the findings might not fully represent the situation of the entire high-risk group. An anonymous survey will also miss part of the information of the respondents. If the survey is combined with individual risk assessment information, the results may be more meaningful. The number of positive patients with free multi-gene detection was small (19), so no significant statistical differences were found in this group, and analysis with a larger sample number may be needed. Finally, "willingness" is the first step; the results of this work show that there is still a gap between true participation in the GT process and a previous profession of willingness to do so.

\section{Conclusions}

This study aimed to understand the awareness and choices of GC and GT among a high-risk of hereditary breast cancer group China. The results indicate that before and after GT, the patients in the high-risk groups have marked differences in the willingness to choose GC and GT. Lack of breast cancer genetic knowledge, misconceptions, cost, fear of adverse effects, policy, and market factors are barriers to acceptance. The doctor needs to fully evaluate the frame of mind of each patient, guide them to interpret the results, and use the positive results for cancer prevention in families. The future improvement of the national policy and GT market might be a benefit to the medical management of hereditary breast cancer in China.

\section{Acknowledgments}

We are particularly grateful to all the patients and their family members who participated in this study. We thank AME Editing Service for its linguistic assistance during the preparation of this manuscript.

Funding: Supported by the Youth Research Foundation (20134Y094) of Shanghai Health Bureau, the Medical Guidance Technology Project (No. 15401932200) of the Municipal Science, the Fundamental Research Funds for the Central Universities (22120180402), Shanghai Science and Technology Committee Projects (15411964800), and Shanghai Municipal Medical and Health Discipline Construction Projects (No. 2017ZZ02015).

\section{Footnote}

Conflicts of Interest: All authors have completed the
ICMJE uniform disclosure form (available at http://dx.doi. org/10.21037/tcr.2019.11.43). The authors have no conflicts of interest to declare.

Ethical Statement: The authors are accountable for all aspects of the work in ensuring that questions related to the accuracy or integrity of any part of the work are appropriately investigated and resolved. The study was conducted in accordance with the Declaration of Helsinki (as revised in 2013). This study has been approved by the Shanghai First Maternity and Infant Hospital and Ethics Committee (IRB number KS1412). Informed consent was obtained from all individual participants included in this study.

Open Access Statement: This is an Open Access article distributed in accordance with the Creative Commons Attribution-NonCommercial-NoDerivs 4.0 International License (CC BY-NC-ND 4.0), which permits the noncommercial replication and distribution of the article with the strict proviso that no changes or edits are made and the original work is properly cited (including links to both the formal publication through the relevant DOI and the license). See: https://creativecommons.org/licenses/by-nc-nd/4.0/.

\section{References}

1. Forouzanfar MH, Foreman KJ, Delossantos AM, et al. Breast and cervical cancer in 187 countries between 1980 and 2010: a systematic analysis. Lancet 2011;378:1461-84.

2. Pharoah PDP, Antoniou A, Bobrow M, et al. Polygenic susceptibility to breast cancer and implications for prevention. Nature Genetics 2002;31:33-6.

3. Foulkes WD. Molecular Origins of Cancer: Inherited Susceptibility to Common Cancers. N Engl J Med 2008;359:2143-53.

4. Buys SS, Sandbach JF, Gammon A, et al. A study of over 35,000 women with breast cancer tested with a 25-gene panel of hereditary cancer genes. Cancer 2017;123:1721-30.

5. Couch FJ, Shimelis H, Hu C, et al. Associations Between Cancer Predisposition Testing Panel Genes and Breast Cancer. Jama Oncol 2017;3:1190-6.

6. Huang Z, Wen W, Zheng Y, et al. Breast cancer incidence and mortality: trends over 40 years among women in Shanghai, China. Ann Oncol 2016;27:1129-34.

7. Cao W, Wang X, Li J-C. Hereditary Breast Cancer in the Han Chinese Population. J Epidemiol 2013;23:75-84.

8. Weitzel JN, Blazer KR, MacDonald DJ, et al. Genetics, 
Genomics, and Cancer Risk Assessment State of the Art and Future Directions in the Era of Personalized Medicine. CA Cancer J Clin 2011;61:327-59.

9. PARP inhibitors arrive in China via new priority review pathway. 2018. https://www.pharmaceutical-technology. com/comment/parp-inhibitors-china/.

10. Kwong A, Ng EKO, Wong CLP, et al. Identification of BRCA1/2 Founder Mutations in Southern Chinese Breast Cancer Patients Using Gene Sequencing and High Resolution DNA Melting Analysis. Plos One 2012;7:e43994.

11. Li WF, Hu Z, Rao NY, et al. The prevalence of BRCA1 and BRCA2 germline mutations in high-risk breast cancer patients of Chinese Han nationality: two recurrent mutations were identified. Breast Cancer Res Treat 2008;110:99-109.

12. Zhang J, Pei R, Pang Z, et al. Prevalence and characterization of BRCA1 and BRCA2 germline mutations in Chinese women with familial breast cancer. Breast Cancer Res Treat 2012;132:421-8.

13. Hall MJ, Forman AD, Pilarski R, et al. Gene Panel Testing for Inherited Cancer Risk. J Natl Compr Canc Netw 2014;12:1339-46.

14. Hall MJ, Obeid E, Daly MB. Multigene Panels to Evaluate Hereditary Cancer Risk: Reckless or Relevant? J Clin Oncol 2016;34:4186-7.

15. Cheng X, Li Z, Sun X, et al. Survey on the knowledge and willingness of genetic counseling and testing in blood relatives of breast cancer patients. Fu Dan Xue Bao Yi Xue Ban 2017;44:312-8.

16. Cheng X, Li Z, Sun X, et al. Knowledge and willingness of breast cancer patients from Shanghai for genetic counseling and gene testing. Zhonghua Yi Xue Yi Chuan Xue Za Zhi 2016;33:589-93.

17. Evans DG, Barwell J, Eccles DM, et al. The Angelina Jolie effect: how high celebrity profile can have a major impact on provision of cancer related services. Breast Cancer Res 2014;16:442.

18. Liede A, Karlan BY, Narod SA. Cancer risks for male carriers of germline mutations in BRCA1 or BRCA2: A review of the literature. J Clin Oncol 2004;22:735-42.

19. Schneider K, Zelley K, Nichols KE, et al. Li-Fraumeni Syndrome - GeneReviews. Gene. 2013.

20. Brooks-Wilson AR, Kaurah P, Suriano G, et al. Germline E-cadherin mutations in hereditary diffuse gastric cancer: assessment of 42 new families and review of genetic screening criteria. J Med Genet 2004;41:508-17.

21. Pilarski R. Cowden Syndrome: A Critical Review of the Clinical Literature. J Genet Couns 2009;18:13-27.

22. Hobert JA, Eng C. PTEN hamartoma tumor syndrome: An overview. Genet Med 2009;11:687-94.

23. Kast K, Rhiem K, Wappenschmidt B, et al. Prevalence of BRCA1/2 germline mutations in 21401 families with breast and ovarian cancer. J Med Genet 2016;53:465-71.

24. Gonzalez-Angulo AM, Timms KM, Liu S, et al. Incidence and Outcome of BRCA Mutations in Unselected Patients with Triple Receptor-Negative Breast Cancer. Clin Cancer Res 2011;17:1082-9.

25. Lee LJ, Alexander B, Schnitt SJ, et al. Clinical Outcome of Triple Negative Breast Cancer in BRCA1 Mutation Carriers and Noncarriers. Cancer 2011;117:3093-100.

26. Paris I, Di Giorgio D, Palluzzi E, et al. Management of BRCA mutation carriers. Transl Cancer Res 2018;7:S329-S38.

27. Gong P, Liang S, Carlton EJ, et al. Urbanisation and health in China. Lancet 2012;379:843-52.

28. Voorwinden JS, Jaspers JPC. Prognostic Factors for Distress After Genetic Testing for Hereditary Cancer. J Genet Couns 2016;25:495-503.
Cite this article as: Cheng X, Gu Z, Sun X, Zhuang Z. Study on the differences of opinions and choices of high-risk breast cancer populations in China before and after genetic testing. Transl Cancer Res 2019;8(8):2893-2905. doi: 10.21037/ tcr.2019.11.43 